\title{
An Updated Review of Hypertensive Emergencies and Urgencies
}

\author{
Katalin Makó, Corina Ureche, Zsuzsanna Jeremiás \\ University of Medicine and Pharmacy, Tîrgu Mureș, Romania
}

\section{ABSTRACT}

A hypertensive crisis is an abrupt and severe rise in the arterial blood pressure (BP) occurring either in patients with known essential or secondary hypertension, or it may develop spontaneously. The most frequent cause for the severe and sudden increase in BP is inadequate dosing or stopping antihypertensive treatment in hypertensive patients. Severe hypertension can be defined as either a hypertensive emergency or an urgency, depending on the existence of organ damage. In hypertensive urgencies, there are no signs of acute end-organ damage, and orally administered drugs might be sufficient. In hypertensive emergencies, signs of acute end-organ damage are present, and in these cases, quickly-acting parenteral drugs must be used. The prompt recognition, assessment, and treatment of hypertensive urgencies and emergencies can decrease target organ damage and mortality. In this review, the definitions and therapeutic recommendations in a hypertensive crisis are presented in the light of the 2017 ACC/AHA Hypertension Guidelines.

Keywords: hypertensive urgencies, hypertensive emergencies, antihypertensive treatment

\section{ARTICLE HISTORY}

Received: April 24, 2018

Accepted: June 9, 2018

\section{CORRESPONDENCE}

\section{Katalin Makó}

Str. Gheorghe Marinescu nr. 38

540139 Tîrgu Mureș, Romania

Tel: +40 265212886

E-mail: makokatalin@yahoo.com

\section{INTRODUCTION}

Arterial hypertension presents a very high incidence and is a frequently encountered clinical situation, affecting nearly 1 billion subjects around the world. The overall sex- and age-adjusted prevalence of hypertension (systolic/diastolic $>130 / 80 \mathrm{mmHg}$ ) is $48 \%$ in males and $43 \%$ in females. ${ }^{1}$

Approximately $1-2 \%$ of subjects with chronic hypertension present acute elevations of blood pressure (BP) that require urgent therapeutic measures, while more than half of subjects presenting in the emergency department with hypertensive crisis have not been compliant to their blood pressure-lowering medication within the preceding days or weeks. ${ }^{2}$

\section{DEFINITIONS}

A hypertensive crisis is an abrupt increase in the systolic BP (SBP) over $180 \mathrm{mmHg}$ and/or elevation of diastolic BP (DBP) over $120 \mathrm{mmHg}$. Based on the existence of acute end-organ damage, a hypertensive crisis can be defined as a hypertensive emergency or urgency. ${ }^{1,3}$

A hypertensive emergency is a critical rise in $\mathrm{BP}$ (of more than $180 / 120 \mathrm{mmHg}$ ) with associated newly developed, progressive or deteriorating target organ injury. Instances of target organ injury comprise hypertensive encephalopathy, intracerebral hemorrhage, acute ischemic stroke, acute myocardial infraction, acute left ventricular failure with pulmonary edema, unstable angina, dissecting aortic aneurysm, acute renal failure, and eclampsia. ${ }^{1,4}$ 
In 2017, Guiga et al. published data on mortality rates in a 670-patient cohort presenting with hypertensive emergencies, and the one-year associated mortality rate was over $79 \%$, while the median survival period was 10.4 months in subjects in which the emergency was untreated. ${ }^{5}$

Subjects with chronic hypertension are regularly able to tolerate significantly higher BP levels than those with previously normal BP values. A review article focusing on the epidemiology, causes, pathophysiology, and prognosis of hypertensive crises, published in 2017 by Ipek et al., revealed that in the evolution and prognosis of hypertensive emergencies, the rate of $\mathrm{BP}$ rise seems to be more important than the actual BP level. ${ }^{6}$ In hypertensive emergencies, fast reduction of $\mathrm{BP}$ is recommended (not necessarily to normal values) in order to avoid and limit additional target organ injury. In adults with a hypertensive emergency, hospital admission and close supervision in an intensive cardiac care facility is recommended, to continuously monitor arterial pressure levels, to assess and treat target organ injury, as well as to administer intravenous antihypertensive medication. Both current European Society of Hypertension (ESH) and European Society of Cardiology (ESC), as well as American College of Cardiology (ACC) and American Heart Association (AHA) guidelines discourage the use of oral antihypertensive treatment for hypertensive emergencies. ${ }^{1,3}$

Hypertensive urgencies are defined as severe BP elevation in chronic hypertensive patients with no acute target organ injury or dysfunction. In the majority of cases, these patients are non-adherent to the prescribed antihypertensive regimen and do not present with clinical or laboratory signs of acute target organ injury. In hypertensive urgencies, BP levels are frequently lowered in the emergency department, with the use of oral medication, and patients do not require hospital admission, but are, however, followed-up in outpatient settings. ${ }^{1,3}$

In 2015, Lagi et al. published a review that analyzed 373 articles directed towards the dissimilarities between hypertensive emergencies and urgencies, while also defining the concept of 'acute hypertension' as being different from 'chronic hypertension'. 7 Acute hypertension may be associated with target organ injury since it is the cause, consequence, or effect of an acute stress. As a result of this review, Lagi et al. proposed a new terminology and a classification system for hypertensive emergencies, as follows: (a) acute primary hypertension, as the cause (a mechanism is set as the first moment of hypertensive disease targeting the heart, retina, brain, and kidney); (b) acute secondary hypertension, as the consequence (a disease modifies the circulating volume and peripheral vascular resistance); and (c) acute associated hypertension without causal relationship (the disease is the effect of an acute stress response). This classification system is based on the pathophysiological mechanism and is a new clinical approach of patients with hypertensive crises. ${ }^{7}$

\section{PATHOPHYSIOLOGY}

The factors that cause the acute severe increase in BP in subjects with hypertensive crises are partially understood. ${ }^{8}$ In a review article, Marik et al. emphasized that the major pathophysiological mechanism is a sudden rise in systemic vascular resistance due the increased production of humoral vasoconstrictors. The subsequent acute and severe elevation of $\mathrm{BP}$ values creates mechanical stress and endothelial damage, thus enhancing vascular permeability, activation of the coagulation cascade, activation of platelets, and fibrin deposition. The vessel damage causes platelet and fibrin deposition, fibrinoid necrosis of the arterioles, and an alteration of the normal self-regulatory properties. ${ }^{8,9}$ The role of the angiotensin-aldosterone system was described in numerous publications as causing further vasoconstriction, stimulating decreased renal perfusion, and lowering tubular sodium concentration. ${ }^{10} \mathrm{~A}$ review by van den Born et al. evaluated whether a hypertensive crisis with advanced retinopathy is related to endothelial dysfunction, platelet activation, thrombin generation, and decreased fibrinolytic activity and concluded that hypertensive crisis leads to an enhanced prothrombotic status due to endothelial dysfunction, platelet activation, and increased thrombin production. ${ }^{11}$ These modifications in the pro- and antithrombotic pathways may add to the already high risk of ischemic and hemorrhagic complications. Pressure natriuresis produces volume depletion, stimulating the release of vasoconstrictor agents from the kidney. The results of these events lead to a vicious cycle of continuous injury producing ischemia and can culminate in end-organ hypoperfusion and dysfunction. . $^{8,10,11}$ Recent studies have also evaluated the role of genetic polymorphisms and have sought to identify susceptibility genes that may lead to the development of arterial hypertension, and also pulmonary arterial hypertension. Several genetic profiles have been identified as factors that influence the blood pressure homeostasis, such as endothelial nitric oxide synthase (eNOS), or polymorphisms encountered in the human angiotensinogen gene (AGT). ${ }^{12,13}$

\section{CLINICAL PRESENTATION}

The clinical manifestation of a hypertensive crisis varies from one individual to another; its clinical signs and 
symptoms are directly connected to the consequent endorgan injury which has been triggered. As Marik et al. presented in a review about the challenges and management of a hypertensive crisis, the most frequent motive for presenting in the emergency department in patients with hypertensive emergencies included acute chest pain $(27 \%)$, shortness of breath (22\%), and neurological impairment (21\%), while the most frequent clinical presentations of hypertensive emergencies were cerebral infarction $(24.5 \%)$, pulmonary edema $(22.5 \%)$, hypertensive encephalopathy $(16.3 \%)$, and congestive heart failure $(12 \%){ }^{8}$ The cardiac manifestations of hypertensive crisis are angina and/or myocardial infarction, congestive heart failure, or pulmonary edema. ${ }^{44,15}$

Manning et al. reviewed the diagnosis and management of specific neurological hypertensive emergencies and described the specific neurologic manifestations of hypertensive encephalopathy as the presence of occipital headache, visual disturbances, vomiting, mental status changes, seizure, and papilledema. ${ }^{16}$ Kidney injury may present as oliguria or any of the typical characteristics of acute renal failure. Gastrointestinal and hematological manifestations may occur as nausea, vomiting, and microangiopathic hemolytic anemia (due to a diffuse arteriolar damage). ${ }^{17}$

\section{CLINICAL APPROACH}

Patients with a hypertensive crisis usually present in the emergency department for newly developed symptoms related to their increased BP values. ${ }^{18}$ The physical examination should be performed quickly in order to recognize signs related to end-organ injury. Proper patient triage is a central phase in the clinical management of hypertensive crises. ${ }^{19} \mathrm{~A}$ quick but complete medical history should be obtained, including pre-existent hypertensive history, current use of antihypertensive treatment, the adherence to the therapeutic schedule, and the time from the last dose. ${ }^{3}$ Assessment of BP values should be performed on both upper limbs, with an appropriately sized cuff. ${ }^{20}$

The physical examination includes assessment of pulses in all limbs, auscultation of the lungs (rales suggestive for pulmonary edema), the heart (murmurs, gallop rhythm), and the renal arteries (bruits), as well as a neurologic and ocular (fundoscopic) check-up. ${ }^{8}$

Laboratory exams (full blood count, creatinine, urea, electrolytes, urine analysis), ECG, chest X-ray, echocardiography, CT (chest/head), MRI should be performed. Based on the physical examination, laboratory examinations, and imaging, the clinician is able to distinguish be- tween a hypertensive emergency and urgency, and to formulate a patient management plan for further diagnostic testing and therapeutic measures. , $^{8,10,21}$

\section{THERAPEUTIC APPROACH OF HYPERTENSIVE CRISIS - "PRIMUM NON NOCERE"}

Hypertensive emergencies require fast measures for decreasing the BP to avoid and limit additional damaging of the target organs. ${ }^{22}$ Patients with a hypertensive emergency require hospital admission and observation in an intensive care unit, with continuous monitoring and further testing for the identification and treatment of target organ damage, as well as administration of intravenous blood pressure-lowering medication., ${ }^{1,3}$ The therapeutic management of hypertensive emergencies is strictly influenced by the subsequent organ damage and starts from no lowering or extremely cautious lowering of BP..$^{15}$

The 2017 ACC/AHA guideline recommends for adults with a critical complication, such as severe preeclampsia, eclampsia, or pheochromocytoma crisis, a reduction of systolic BP to less than $140 \mathrm{mmHg}$ during the first hour, while in aortic dissection to less than $120 \mathrm{mmHg}$. For subjects with no critical condition, systolic BP is reduced by less than $25 \%$ in the first hour, then the target value is carefully achieved in the next $24-48$ hours. ${ }^{1}$

In contrast, in patients with hypertensive urgencies without reason for referral to the emergency department or for hospitalization, blood pressure values can be typically lowered in the emergency room, with oral medication (reinstitution or intensification of antihypertensive therapy)., ${ }^{1,3,24}$

Several antihypertensive drugs from different pharmacological categories are accessible for managing hypertensive emergencies, but there is no evidence which drug class administered as first-line therapy provides more benefits. ${ }^{25,26}$

A Cochrane systematic review on therapeutic measures applied in hypertensive emergencies, which studied 15 randomized controlled trials and 869 subjects treated with seven drug classes, did not find sufficient data for defining which antihypertensive medication is the most efficient in treating hypertensive emergencies and decreasing mortality. ${ }^{25}$ Randomized clinical trials are needed to investigate mortality and outcomes in patients with hypertensive emergencies treated with different antihypertensive drugs, and rate and grade BP lowering in a hypertensive emergency.

According to actual guidelines (ESH/ESC and ACC/AHA), in hypertensive emergencies, continuous intravenous ad- 
ministration of short-acting antihypertensive therapies is recommended. ${ }^{1,23}$ Choosing an antihypertensive class should be grounded on its pharmacological characteristics, the degree and advancement of target organ damage, the required rate of $\mathrm{BP}$ reduction, and comorbidities. ${ }^{27}$ The dosage of these agents must be titrable for a controllable antihypertensive effect and for the prevention of further target organ damage, because an excessive and rapid lowering of BP values may cause coronary, cerebral, or renal ischemia. ${ }^{28,29}$

\section{INTRAVENOUS ANTIHYPERTENSIVE MEDICATION IN HYPERTENSIVE EMERGENCIES}

\section{CALCIUM CHANNEL BLOCKERS - DIHYDROPYRIDINES}

Nicardipine is a second-generation short-acting dihydropyridine-type calcium-channel blocker. The onset of action of intravenously administered nicardipine is from 5 to 15 minutes, the duration of action is 4 to 6 hours..$^{30}$ It is characterized by a high vascular selectivity and strong coronary and cerebral vasodilator activity. CLUE was the first randomized clinical trial that comparatively analyzed nicardipine infusion versus bolus labetalol in the management of acute hypertension. The trial showed that subjects who were administered nicardipine were more expected to have their BP controlled compared to those who were treated with labetalol (OR 2.73, 95\% CI 1.1 to 6.7). ${ }^{31}$ Nicardipine may have unique benefits in cerebrovascular disease based on its pharmacologic profile. It crosses the blood-brain barrier and produces relaxation of cerebrovascular smooth muscle. At the acidic $\mathrm{pH}$ of ischemic cerebral tissue, nicardipine is rapidly accumulated in the ischemic tissue, producing localized vasodilation. Although nicardipine is a cerebral vasodilator, it dilates small-resistance arterioles, so there are no significant changes in intracranial volume or pressure. Komura et al. published a literature review and the first two documented cases of nicardipine used as an intravenous bolus dose in an acute ischemic stroke and a hemorrhagic stroke subject, respectively. They concluded that nicardipine as an intravenous medication is effective and safe in these situations. ${ }^{32}$

The recommended initial infusion rate of nicardipine is $5 \mathrm{mg} / \mathrm{h}$, which may be increased every 5 minutes by $2.5 \mathrm{mg} / \mathrm{h}$, up to a maximum of $15 \mathrm{mg} / \mathrm{h}$ until the chosen BP reduction is attained. There is no required dose modification in case of elderly patients, and there is no dependence on the patient's weight. Nicardipine is contraindicated in advanced aortic stenosis. ${ }^{1,3}$
Clevidipine is a third-generation dihydropyridine calcium-channel blocker with ultrashort action, with a highly selective arteriolar vasodilator effect. 33,34 This effect is due to a selective inhibition of the influx of calcium through the L-type channel, producing vasodilatation of the small arteries and reduction of peripheral vascular resistance, thus reducing the afterload without affecting cardiac filling pressures or reflex tachycardia. ${ }^{8}$ Clevidipine also protects against organ reperfusion injury and increases the bioavailability of nitric oxide via antioxidative actions. Clevidipine may reduce the severity of myocardial ischemia, preserve coronary endothelial function, reduce infarct size, and maintain renal function by preserving splanchnic blood flow. ${ }^{35,36}$ The ECLIPSE trial was conducted for a comparative analysis between the safety and efficacy of clevidipine with nitroglycerin, sodium nitroprusside, and nicardipine in the treatment of perioperative acute hypertension in patients undergoing cardiac surgery. The results showed that mortality was significantly higher for nitroprusside-treated subjects compared with the clevidipine-treated arm ( $\mathrm{p}=$ 0.04). Clevidipine was more effective than nitroglycerine $(\mathrm{p}=0.0006)$ or nitroprusside $(\mathrm{p}=0.003)$ in maintaining BP within the prespecified interval. Clevidipine was comparable to nicardipine in keeping patients within the prespecified BP range. There was no difference between those treated with clevidipine and the other treatment groups regarding the rate of major adverse cardiovascular events including myocardial infarction, stroke, or renal dysfunction. ${ }^{35}$

The initial dose recommended for clevidipine is 1 to $2 \mathrm{mg} / \mathrm{h}$ in intravenous infusion, which can initially be doubled at short ( $90 \mathrm{sec}$ ) intervals. The anticipated therapeutic response for most individuals ensues at doses of 4 to $6 \mathrm{mg} / \mathrm{h}$. Subjects with severe hypertension may need doses up to $32 \mathrm{mg} / \mathrm{h}$. Clevidipine is contraindicated in subjects who are allergic to soy- and egg-related products, and in patients with defective lipid metabolism. Dose adjustment is required for the elderly. ${ }^{1,8}$

\section{NITRIC OXIDE-DERIVED VASODILATORS}

Sodium nitroprusside is a prodrug that reacts with sulfhydryl groups on erythrocytes, albumin, and other serum proteins to release nitric oxide (NO). NO reduces the vascular tone in small muscular arteries, via stimulation of the guanyl-cyclase-cyclic GMP pathway. In 2014, in a review by Hottinger et al., nitroprusside was described as the most frequently used intravenous drug in hypertensive crises in the emergency department or intensive care 
facilities, followed by labetalol ( $48 \%$ of cases), nicardipine $(15 \%)$, and hydralazine (15\%). ${ }^{36}$

Nitroprusside decreases cerebral vascular resistance and impairs brain and myocardial tissue oxygenation. Early animal studies described that nitroprusside causes vasodilation and maintains cerebral blood flow inhibiting the vasospasm that occurs immediately after a subarachnoid hemorrhage. ${ }^{36} \mathrm{~A}$ study by Immink et al. evaluated the effect of labetalol versus nitroprusside in the treatment of malignant hypertension, as well as their influence of cerebral circulation and their hemodynamic effects. ${ }^{37}$ They concluded that sodium nitroprusside lowered vascular resistance and not cerebral vascular resistance, thus stimulating a redirecting of the blood flow towards territories with low vascular resistance rather than towards the brain circulation.

The ECLIPSE trial compared nitroprusside with clevidipine and nicardipine, showing results of similar efficacy on $\mathrm{BP}$ reduction and small differences in adverse effects. ${ }^{35}$

The initial dose of sodium nitroprusside is $0.3-0.5 \mathrm{mcg} /$ $\mathrm{kg} / \mathrm{min}$ intravenously, with increases in increments of $0.5 \mu \mathrm{g} / \mathrm{kg} / \mathrm{min}$ to reach the established BP goal values, with an instantaneous effect and duration of effect lasting 2-3 minutes. The maximum dose is $10 \mathrm{mcg} / \mathrm{kg} / \mathrm{min}$ administered in as short a time as possible. A main concern related to the use of sodium nitroprusside is the accumulation of toxic metabolites. ${ }^{36}$ Cyanide toxicity results in cellular hypoxia and is clinically manifested by irreversible neurologic changes and cardiac arrest. ${ }^{1}$ For infusion rates $\geq 4-10 \mathrm{mcg} / \mathrm{kg} / \mathrm{min}$ or duration $>30 \mathrm{~min}$, thiosulfate can be co-administered to prevent cyanide toxicity. Intra-arterial BP measuring is also recommended to prevent overdosing. Dose adjustment is needed for elderly patients. ${ }^{3,8,21}$ The current AHA/ACC guidelines recommend the use of nitroprusside when treating acute hypertension with a target SBP under $180 \mathrm{mmHg}$ in subjects with intracranial bleeding. ${ }^{1}$

Nitroglycerin is a powerful venous vasodilator that only acts on the arterial vascular tonus at increased doses and lowers BP by decreasing venous return (preload) and cardiac output. ${ }^{8}$ The initial recommended dose is $5 \mathrm{mcg} / \mathrm{min}$, which can be increased in increments of $5 \mathrm{mcg} / \mathrm{min}$ every 3-5 min to a maximum of $20 \mathrm{mcg} / \mathrm{min}$. Tolerance to the hemodynamic effects of nitroglycerin may limit its clinical usefulness. ${ }^{21}$ In the ECLIPSE trial, clevidipine was more effective compared with nitroglycerine $(p=0.0006)$ in sustaining BP in the target range. There was no difference between the nitroglycerin group and the clevidipine- and nitroprusside-treated patients regarding the rate of myocardial infarction, stroke, or renal failure. ${ }^{35}$
Headache is the most common adverse effect of nitroglycerin treatment, and another potential side effect is low blood pressure with reflex tachycardia, which can be aggravated by volume depletion typically used in hypertensive emergencies. ${ }^{38}$ Another undesirable effect of nitroglycerin is compromised cerebral and renal perfusion, therefore, its use is recommended only in subjects presenting an acute coronary syndrome or acute pulmonary edema and is contraindicated in patients with volume depletion. ${ }^{1}$

\section{DIRECT VASODILATORS}

Hydralazine inhibits calcium release from the sarcoplasmic reticulum in arteriolar smooth muscle fibers, thus leading to peripheral vasodilation, which in turn decreases cardiac afterload and improves left ventricular function in subjects with heart failure. ${ }^{39}$ However, hydralazine is not regularly used as a first-line therapy in the treatment of hypertensive emergencies as it presents prolonged and unpredictable blood pressure-lowering properties. ${ }^{1}$

\section{ADRENERGIC BLOCKERS}

Esmolol is an ultrashort-acting cardiac beta-1 receptor blocker, with an ultra-rapid onset of action (within 60 seconds) and duration of action of 10 to 20 minutes. The loading dose is $500-1000 \mathrm{\mu g} / \mathrm{kg} / \mathrm{min}$ administered in 1 minute, with a subsequent infusion of 50 to $200 \mu \mathrm{g} / \mathrm{kg} / \mathrm{min}$ if necessary. Esmolol is an appropriate pharmacological agent for situations of increased cardiac output, heart rate, or blood pressure such as perioperative hypertension. ${ }^{1,21}$ Landoni et al. conducted a meta-analysis on the clinical effects of esmolol in noncardiac surgery, which included 1,765 subjects from 32 randomized clinical trials. The results showed that the esmolol group presented significantly lower rates of myocardial ischemic events and less episodes of hypotension or bradycardia compared to controls. ${ }^{29}$ Contraindications for the use of esmolol comprise subjects under concomitant beta-blocker therapy, low heart rate, acute or decompensated heart failure. Continuous heart rate monitoring is recommended for patients receiving esmolol in order to promptly recognize severe bradycardia and notice heart failure symptoms, as they may worsen. ${ }^{1}$ The metabolism of esmolol is not dependent on kidney or liver function, and it is considered "an ideal ß-adrenergic antagonist" in critically ill subjects due to its pharmacokinetic properties. ${ }^{28,29}$

Labetalol, a non-selective alpha- and beta-adrenergic receptor blocker, presents vasodilator properties in the pe- 
ripheral vasculature by inhibiting adrenergic stimulation in the vascular smooth muscle cells and endothelial function. Through its beta-blockade function, it acts in the bronchial and vascular smooth muscle fibers as well. The overall systemic effect of labetalol is to reduce systolic and diastolic arterial pressure, resting and exercise cardiac frequency, and cardiac output.7,21 A systematic review published by Peacock et al. included comparative studies between the effects of labetalol and nicardipine in hypertensive crisis subjects, with various associated clinical conditions such as stroke, critical care, perioperative status, pediatrics, and pregnancy. Their results revealed that there is equivalent safety and efficacy in the use of labetalol or nicardipine, despite the fact that nicardipine is able to provide a slightly more consistent BP control. ${ }^{31}$ The initial dose of labetalol is $0.3-1.0 \mathrm{mg} / \mathrm{kg}$ (maximum $20 \mathrm{mg}$ ) in slow intravenous injection every 10 minutes, or $0.4-1.0 \mathrm{mg} / \mathrm{kg} / \mathrm{h}$ intravenous infusion up to $3 \mathrm{mg} / \mathrm{kg} / \mathrm{h}$. The cumulative dose is $300 \mathrm{mg}$. This dose can be repeated every 4-6 hours. Labetalol is contraindicated in bronchial asthma and chronic obstructive pulmonary disease. Due to its negative inotrope and chronotropic effects, it should not be given in subjects with heart failure and with second- or third-degree atrioventricular block or bradycardia. ${ }^{1,28}$

Phentolamine is a competitive $\alpha$-adrenergic receptor antagonist with greater affinity for the $\alpha 1$ - than $\alpha 2$-receptor subtype, producing a significant reduction in systemic vascular resistance and an increase in cardiac output, myocardial oxygen demand, and heart rate. ${ }^{7}$ Phentolamine is recommended in hypertensive emergencies caused by excessive catecholamine release including pheochromocytoma, clonidine withdrawal, cocaine toxicity, interactions between monoamine oxidase inhibitors and other drugs or food, as well as amphetamine overdose. ${ }^{1,28}$ The recommended dose is an intravenous bolus of $5 \mathrm{mg}$, and additional bolus doses can be administered every 10 minutes, as needed to lower BP to the target value. Adverse effects include reflex tachycardia and fluid retention, and it is contraindicated in myocardial ischemia. ${ }^{1,3}$

\section{DOPAMINE 1-RECEPTOR SELECTIVE AGONIST}

Fenoldopam is an agonist for D1-like dopamine receptors, with moderate affinity to $\alpha 2$-adrenergic receptors. As a D1-dopamine receptor agonist is a rapid-acting vasodilator, it leads to a decreased peripheral vascular resistance and increased blood flow to the kidneys, while also having minimal diuretic (natriuretic) effects..$^{40}$ This favorable effect on renal perfusion was confirmed by a meta-analysis by Gillies et al., on 6 randomized controlled trials, which found that patients who had received fenoldopam treatment in the perioperative period, presented a significantly lower rate of acute kidney injury following a major surgical intervention..$^{40}$ Fenoldopam is contraindicated in subjects at high risk of conditions with increased intraocular (glaucoma) or intracranial pressure and those with sulfite allergy. ${ }^{1}$ Adverse effects are hypotension and severe headache. ${ }^{3}$

\section{ACE INHIBITORS}

Enalaprilat is an angiotensin-converting enzyme (ACE) inhibitor with vasodilator properties due to the decreased production of angiotensin II and increased levels of bradykinin. In patients with hypertension, the administration of enalaprilat produces the reduction of total peripheral resistance with slight influence on the heart rate or cardiac output. ${ }^{41}$ In a retrospective cohort study by Avaz et al., the administration of bolus intravenous enalaprilat was evaluated when administered to hypertensive patients with acute heart failure, resulting in a substantial reduction in systolic BP. The treatment was well tolerated, with limited hypotension and no adverse effect on kidney function. ${ }^{41}$ The recommended dose of enalaprilat is $1.25 \mathrm{mg}$ administered over a 5-minute period. ${ }^{1}$ The onset of enalaprilat effect occurs in 15-30 minutes, the duration of its action is $12-24$ hours, and it produces an unpredictability of $\mathrm{BP}$ response. In contrast to shorter-acting vasodilators used for the treatment of hypertensive emergency, the dosage of enalaprilat is not easily adjusted. The degree of BP lowering associated with enalaprilat depends on the pretreatment concentration of angiotensin II and plasma renin activity, thus being more efficient in individuals with high renin levels. ${ }^{1,7}$ Enalaprilat should be avoided in patients with acute myocardial infarction and is contraindicated in those with bilateral renal artery stenosis, or in pregnancy.,21

Compared to ESH recommendations, in the 2017 ACC/ AHA guidelines the use of the loop-diuretic furosemide is not recommended as a first-line therapy in the treatment of hypertensive emergencies. ${ }^{3,23}$ Volume depletion is frequent in subjects with hypertensive emergencies, therefore, the administration of a diuretic alone or combined with an antihypertensive medication can trigger a serious and dangerous reduction in BP and produce an increased reflex vasoconstriction with further damage of the target organs. Furosemide must not be used except when it is specifically needed for volume overload, for instance in renal parenchymal disease. ${ }^{1,21}$

Urapidil, an alpha-blocker, has also been recommended by the ESH in the treatment of hypertensive emergencies and does not appear as possible first-line medication for 
this pathology in the 2017 ACC/AHA guidelines. ${ }^{1,3}$ Urapidil is an alpha-blocker with supplementary effects on the central nervous system (activates 5-HT1A receptors) and induces vasodilatation without tachycardia. The recommended dose of urapidil is $25-50 \mathrm{mg}$ in bolus, the onset of action is $3-4$ minutes with a duration of $8-12$ hours. ${ }^{3}$

\section{THE TREATMENT OF HYPERTENSIVE EMERGENCIES IN PATIENTS WITH SPECIFIC COMORBIDITIES}

Subjects with hypertensive emergencies present with target organ damage, which includes acute aortic dissection, acute pulmonary edema, acute coronary syndromes, acute kidney injury, eclampsia or pre-eclampsia, perioperative hypertension, states with excessive catecholamine discharge, acute intracerebral hemorrhage, or acute ischemic stroke. ${ }^{1,3}$ Such individuals require immediate and efficient therapeutic management, with intravenous medication for a safe decrease in blood pressure, with protective effects on the function of target organs, which improves symptoms and decreases the rate of complications, while also improving clinical outcomes. ${ }^{42}$

\section{ACUTE AORTIC DISSECTION}

If left untreated, this hypertensive emergency has a oneyear mortality rate of $70-90 \%$ and a 5-year mortality rate of nearly $100 \%$. In a review article focused on the management of aortic dissection with hypertensive emergency, Gupta et al. described a decrease in the 1-year and 5-year mortality rates of $25 \%$ and $50 \%$ respectively, through an adequate control of BP.43

The medication of choice in the management of acute aortic dissection is intravenous labetalol or esmolol. ${ }^{1}$ Fast and immediate lowering of $\mathrm{BP}$ within 20 minutes is needed for these patients, the target value being below $120 \mathrm{mmHg}$ for systolic BP values. If the BP is still elevated after beta blocker administration, a nitrate-based vasodilator, such as nitroglycerin or nitroprusside, is recommended. Beta blockade should precede the infusion of vasodilator therapy if needed, in order to control BP and to avoid reflex tachycardia. 43

\section{ACUTE PULMONARY EDEMA}

The medication of choice for the management of malignant hypertension associated with acute pulmonary edema includes clevidipine, or nitrate-based vasodilators such as nitroglycerin or nitroprusside. ${ }^{1}$ The $\mathrm{BP}$ in these patients should be decreased in the first few minutes and up to one hour, with approximately $20-25 \%$, followed by a decrease to $160 / 100 \mathrm{mmHg}$ in the subsequent 2-6 hours, with the goal of reaching normal arterial pressure values in the next 24-48 hours. Beta-1 receptor antagonists should not be used in the treatment of acute pulmonary edema, as higher doses can further decrease the already impaired respiratory function, due to beta-2 adrenergic stimulation and consequent bronchoconstriction. ${ }^{44}$ PRONTO was a randomized, active control trial of clevidipine versus standard-of-care (SOC) intravenous antihypertensive therapy in patients with acute heart failure in the emergency room. More clevidipine patients (71\%) reached the prespecified target BP range than those receiving standard care $(37 \% ; \mathrm{p}=0.002)$. Serious adverse events (24\% vs. $19 \%$ ) and 30-day mortality were similar between the clevidipine and the SOC group. 44

\section{ACUTE CORONARY SYNDROMES}

Antihypertensive therapy in acute coronary syndrome (ACS) patients is comprised of multiple pharmacological agents. ${ }^{45}$ The guidelines of the ACC/AHA recommend for patients with hypertensive emergency and ACS (acute myocardial infarction with or without ST segment elevation and unstable angina) treatment with intravenous esmolol, labetalol, nicardipine, or nitroglycerin.1 The target blood BP in hemodynamically stable patients with ACS is $<140 / 90 \mathrm{mmHg}$. It is recommended to maintain the diastolic BP over $60 \mathrm{mmHg}$ to prevent the decrease of coronary perfusion and aggravation of myocardial ischemia below this value. ${ }^{46}$ Beta blockers are contraindicated in moderate to severe left ventricular failure, hypotension (systolic BP $<100 \mathrm{mmHg}$ ), bradycardia $(<60 \mathrm{bpm})$, second- or third-degree atrioventricular block, and reactive airways disorders. ${ }^{1,21}$

\section{ACUTE RENAL INJURY}

Individuals presenting a hypertensive emergency in association with acute kidney injury are electively treated with clevidipine, fenoldopam. or nicardipine. ${ }^{1}$ Clevidipine is a safe therapeutic choice due to its tissular metabolization properties, which are independent of renal function. ${ }^{47}$ VELOCITY, a multicentric trial that included 126 patients with severe hypertension, aimed to evaluate the safety and efficacy of clevidipine in patients with kidney failure. The results were the first to prove that clevidipine is a safe alternative for kidney failure patients with severely increased blood pressure, and continuous prolonged intra- 
venous infusion of clevidipine allowed a successful switch to oral antihypertensive treatment.

As we presented above, fenoldopam lowers the peripheral vascular resistance and concomitantly stimulates renal perfusion in subjects with non-impaired kidney function, as well as in those with chronic renal insufficiency. Data from experimental acute kidney injury models advocate that it also has anti-inflammatory properties, independent of its vasodilator actions..$^{40,48}$

\section{ECLAMPSIA OR PREECLAMPSIA}

The target systolic blood pressure in eclamptic or preeclamptic women is below $140 \mathrm{mmHg}$ within the first hour. ${ }^{49}$ Hydralazine, labetalol, and nicardipine are the medication of choice in the treatment of this hypertensive emergency. ${ }^{1,38,47}$ Inhibitors of the renin-angiotensin-aldosterone system, as well as nitroprusside are contraindicated in the treatment of hypertensive emergencies occurring in pregnant women. If the thrombocyte number is less than 100.000 cells $/ \mathrm{mm}^{3}$, the BP should be maintained below 150/100 mm Hg. ${ }^{1,49}$

\section{PERIOPERATIVE HYPERTENSION}

Perioperative hypertension is defined as a BP of 160/90 $\mathrm{mmHg}$ or higher or an SBP elevation of at least $20 \%$ of the preoperative value, that persists for longer than 15 minutes. Intraoperative hypertension is most frequently encountered during anesthesia induction and airway manipulation. ${ }^{50}$ Intravenous administration of clevidipine, esmolol, nicardipine, or nitroglycerin are the elective therapeutic management option for this hypertensive emergency. ${ }^{1,51,52}$

\section{STATES WITH EXCESSIVE CATECHOLAMINE DISCHARGE}

Excessive catecholamine discharge, including pheochromocytoma or a hyperadrenergic status, are treated with intravenous infusion of phentolamine, nicardipine, or clevidipine. ${ }^{1}$ The Endocrine Society Clinical Practice Guideline for Pheochromocytoma endorses decreasing the systolic BP to below $140 \mathrm{mmHg}$ within the first 60 minutes by administering intravenous phentolamine as needed to achieve the target BP. Beta-adrenergic antagonists should be avoided before administering phentolamine. ${ }^{53}$

\section{ACUTE INTRACEREBRAL HEMORRHAGE}

For acute intracranial bleeding, the ideal pharmacological agents include labetalol, nicardipine, or esmolol. ${ }^{1}$ Sodium nitroprusside and hydralazine should be avoided. In adults with acute intracerebral hemorrhage who present with a systolic BP above $220 \mathrm{mmHg}$, intravenous antihypertensive treatment and close BP monitoring is recommended. In subjects with spontaneous intracerebral hemorrhage presenting within the first 6 hours from onset, with a systolic BP ranging from 150 to $220 \mathrm{mmHg}$, immediate lowering of systolic BP below $140 \mathrm{mmHg}$ can be potentially harmful. ${ }^{54-56}$

The safety and feasibility of intensive BP lowering in patients with acute hemorrhagic stroke was investigated by two pilot, prospective, randomized trials. The INTERACT1 was an international, blinded end-point trial, which enrolled 404 subjects with acute intracerebral hemorrhage and elevated systolic BP levels between 150-220 mmHg within 6 hours from onset. The patients were randomized to either intensive BP management (SBP target $<140$ $\mathrm{mmHg}$ ) or guideline-based management of BP (SBP target $<180 \mathrm{mmHg}$ ). The study aimed to evaluate the safety, efficacy, and clinical outcomes (death or disability) of treatment at 90 days. The intensive BP reduction attenuated proportional hematoma growth at 24 hours (not significantly though, $\mathrm{p}=0.06$ ), and the rates of serious adverse events, neurological deterioration, and poor clinical outcome did not differ significantly between the two groups. The major concern of this treatment strategy was a perihematomal ischemia due to the further reduction of the already compromised cerebral blood flow (produced by the compression of small arteries by the hematoma and the associated edema). The ICH-ADAPT trial sought to analyze the impact of aggressive BP lowering on cerebral blood flow and provided strong arguments against this concern. The results of this study revealed that intensive BP treatment was not related to impaired perihematomal cerebral blood flow compared to the SOC group. ${ }^{57}$ This finding further supports the safety of early BP lowering in acute intracerebral bleeding. However, it also indicates that excessive BP treatment has no effect on diminishing the perihematomal edema.

\section{ACUTE ISCHEMIC STROKE}

For acute ischemic stroke, the ideal therapeutic agents are labetalol and nicardipine. ${ }^{1}$ In subjects with acute $(<72 \mathrm{~h}$ from symptom onset) ischemic stroke and increased arterial pressure, who undergo thrombolytic treatment, the target BP value is below $180 / 105 \mathrm{mmHg}$, which should be maintained for a minimum of 24 hours after thrombolysis, with the use of a slow-acting hypertensive medication. ${ }^{1,58,59}$ In patients with BP over 220/120 $\mathrm{mmHg}$, who are 
not eligible to receive intravenous alteplase or endovascular treatment, without co-morbidities, but who require acute BP lowering therapy, the benefits of antihypertensive treatment within the first 48 to 72 hours is uncertain. A lowering of BP by $15 \%$ within the first 24 hours from onset of the acute cerebrovascular event might be useful. $1,58,59$ However, individuals with $\mathrm{BP}<220 / 120 \mathrm{mmHg}$ gain no benefits from initiating or reinitiating antihypertensive treatment in the first 48-72 hours after the stroke, unless they are eligible for fibrinolysis. ${ }^{1,58,59}$

In the CATIS trial, which included 4,071 hypertensive patients with non-thrombolysed ischemic stroke within 48 hours of onset, subjects were randomly assigned to either receive antihypertensive treatment (to achieve a BP of under $140 / 90 \mathrm{mmHg}$ within 7 days) or to discontinue all antihypertensive medications (control) during hospitalization. The conclusion of this trial was that in subjects with acute ischemic stroke, the administration of BP-lowering medications, compared with the absence of hypertensive drugs, did not reduce the risk of death and major disability at 14 days or at hospital discharge. ${ }^{59}$

\section{TREATMENT OF HYPERTENSIVE URGENCIES}

Hypertensive urgencies represent the severe elevation of BP (>180/120 mmHg) but without signs of acute target organ damage. In these individuals, BP should be lowered progressively, over a period of 24 to 48 hours, and for orally administered antihypertensive drugs, a close follow-up in outpatient settings is strongly recommended. BP lowering should be gradual, as there is no confirmed benefit from a rapid decrease in $\mathrm{BP}$ in patients without any symptoms of acute target organ damage. Also, a rapid fall in BP could produce important organ damage. ${ }^{3}$

The recommended oral medication for hypertensive urgencies includes captopril (12.5-25 mg p.o.), labetalol (200-400 mg p.o.), amlodipine (5-10 mg p.o.), felodipine (5-10 mg p.o.), isradipine (5-10 $\mathrm{mg}$ p.o.), and prazosin (1-2 mg p.o.). ${ }^{3}$ As we mentioned above, loop diuretics such as furosemide should be avoided if there is no specific indication for conditions that present volume overload, as for example in renal parenchymal disease. ${ }^{9}$

Oral (sublingual) nifedipine is a frequently used pharmacological agent for the treatment of hypertensive urgencies. After nifedipine administration, within the first 5-10 minutes after ingestion, an important decrease in the arterial pressure is registered, with a maximum effect in 30-60 minutes and a duration of circa 6-8 hours. Severe and uncontrolled decrease in BP caused by nifedipine may trigger cerebral and myocardial ischemic events. Due to such serious adverse effects, as well as the lack of scientific evidence to confirm its benefits, nifedipine is not recommended in treating hypertensive urgencies. ${ }^{3,9}$

\section{CONCLUSIONS}

Patients presenting in the emergency department with severely increased arterial pressure require careful examination for signs and symptoms of target organ damage. A proper initial assessment in needed in order to further direct patient management, for identification and treatment of possible life threatening complications such as aortic dissection, acute coronary syndromes, acute heart failure or cerebrovascular events. The prompt recognition, assessment and treatment of hypertensive urgencies and emergencies can decrease organ damage and mortality.

\section{CONFLICT OF INTEREST}

Nothing to disclose.

\section{REFERENCES}

1. Whelton PK, Carey RM, Aronow WS, et al. 2017 ACC/AHA/ AAPA/ABC/ACPM/AGS/APhA/ASH/ASPC/NMA/PCNA Guideline for the Prevention, Detection, Evaluation, and Management of High BP in Adults: A Report of the American College of Cardiology/American Heart Association Task Force on Clinical Practice Guidelines. Hypertension. 2018;71:12691324. doi: 10.1161/HYP.0000000000000066.

2. Janke AT, McNaughton CD, Brody AM, Welch RD, Levy PD. Trends in the incidence of hypertensive emergencies in US emergency departments from 2006 to 2013. J Am Heart Assoc. 2016;5:e004511. doi:10.1161/JAHA.116.004511.

3. Vaughan CJ, Delanty N. Hypertensive emergencies. Lancet. 2000;356:411-417.

4. Varounis C, Katsi V, Nihoyannopoulos P, Lekakis J, Tousoulis D. Cardiovascular Hypertensive Crisis: Recent Evidence and Review of the Literature. Frontiers in Cardiovascular Medicine. 2016;3:51. doi:10.3389/fcvm.2016.00051.

5. Guiga H, Decroux C, Michelet P, et al. Hospital and out-ofhospital mortality in 670 hypertensive emergencies and urgencies. J Clin Hypertens. 2017;19:1137-1142. doi: 10.1111/ jch.13083.

6. Ipek E, Oktay AA, Krim SR. Hypertensive crisis: an update on clinical approach and management. Curr Opin Cardiol. 2017;32:397-406. doi: 10.1097/HCO.000000000000039.

7. Lagi A, S Cencetti. Hypertensive emergencies: a new clinical approach. Clinical Hypertension. 2015;21:20. doi.org/10.1186/ s40885-015-0027-4.

8. Marik PE, Varon J. Hypertensive crises: challenges and management. Chest. 2007;131:1949-1962. doi: 10.1378/ chest.06-2490.

9. Derhaschnig U, Testori C, Riedmueller E, Aschauer S, Wolzt M, Jilma B. Hypertensive emergencies are associated with elevated markers of inflammation, coagulation, platelet 
activation and fibrinolysis. Journal of Human Hypertension. 2013;27:368-373. doi:10.1038/jhh.2012.53.

10. Taylor DA. Hypertensive crisis: a review of pathophysiology and treatment. Crit Care Nurs Clin North Am. 2015;27:439447. doi: 10.1016/j.cnc.2015.08.003.

11. van den Born BJ, Löwenberg EC, van der Hoeven NV, et al. Endothelial dysfunction, platelet activation, thrombogenesis and fibrinolysis in patients with hypertensivecrisis. J Hypertens. 2011;29:922-927. doi:10.1097/HJH.ob013e328345023d.

12. Gatti R, Santos P, Angela AS, Marangoni K, Araujo M, Goulart L. The interaction of AGT and NOS3 gene polymorphisms with conventional risk factors increases predisposition to hypertension. Journal of Renin-Angiotensin-Aldosterone System. 2012;14:360-368. doi: 10.1177/1470320312452027.

13. Toganel R, Muntean I, Duicu C, Fagarasan A, Gozar L, Banescu $\mathrm{C}$. The role of eNOS and AGT gene polymorphisms in secondary pulmonary arterial hypertension in Romanian children with congenital heart disease. Revista Romana de Medicina de Laborator. 2013;21:267-274. doi: https://doi.org/10.2478/ rrlm-2013-0031.

14. Varon J, Marik PE. Clinical review: The management of hypertensive crises. Critical Care. 2003;7:374-384. doi: 10.1186/cc2351.

15. Papadopoulos DP, Sanidas EA, Viniou NA, et al. Cardiovascular hypertensive emergencies. Curr Hypertens Rep. 2015;17:5. doi: 10.1007/s11906-014-0515-z.

16. Manning L, Robinson TG, Anderson CS. Control of BP in hypertensive neurological emergencies. Curr Hypertens Rep. 2014;16:436. doi: 10.1007/s11906-014-0436-x.

17. Keddis MT, Garovic VD. 38-Year-Old Woman with Hypertension, Headaches, and Abdominal Bruit. Mayo Clinic Proceedings. 2010;85:674-677. doi:10.4065/mcp.2009.0330.

18. Elliott WJ. Clinical features and management of selected hypertensive emergencies. J Clin Hypertens (Greenwich). 2004; 6:587-592.

19. Pergolini MS. The management of hypertensive crises: a clinical review. Clin Ter. 2009;160:151-157.

20. Varon J. The diagnosis and treatment of hypertensive crises. Postgrad Med. 2009;121:5-13. doi:10.3810/pgm.2009.01.1950.

21. Aronow WS. Treatment of hypertensive emergencies. Ann Transl Med. 2017;5:S5. doi: 10.21037/atm.2017.03.34.

22. Polly DM, Paciullo CA, Hatfield CJ. Management of hypertensive emergency and urgency. Adv Emerg Nurs J. 2011;33:127-136. doi: 10.1097/TME.ob013e318217a564.

23. Mancia G, Fagard R, Narkiewicz K, et al. 2013 ESH/ESC Guidelines for the management of arterial hypertension: The Task Force for the management of arterial hypertension of the European Society of Hypertension (ESH) and of the European Society of Cardiology (ESC). J Hypertens. 2013;31:1281-1357. doi: 10.1097/01.hjh.0000431740.32696.cc.

24. Grassi D, O'Flaherty M, Pellizzari M, et al. Hypertensive urgencies in the emergency department: evaluating blood pressure response to rest and to antihypertensive drugs with different profiles. J Clin Hypertens (Greenwich). 2008;10:662667. doi: 10.1111/j.1751-7176.2008.00001.x.

25. Perez MI, Musini VM. Pharmacological interventions for hypertensive emergencies: a Cochrane systematic review. J Hum Hypertens. 2008;22:596-607. doi: 10.1038/jhh.2008.25.

26. Mansoor GA, Frishman WH. Comprehensive management of hypertensive emergencies and urgencies. Heart Dis. 2002;4:358-371. doi: 10.1097/01.HDX.0000038720.26409.41.
27. Fenves AZ, Ram CV. Drug treatment of hypertensive urgencies and emergencies. Semin Nephrol. 2005;25:272-280. doi: 10.1016/j.semnephrol.2005.02.011.

28. Rhoney D, Peacock WF. Intravenous therapy for hypertensive emergencies, part 1. Am J Health Syst Pharm. 2009; 66:13431352. Am J Health Syst Pharm. 20091;66:1343-1352. doi: 10.2146/ajhp080348.p1.

29. Landoni G, Turi S, Biondi-Zoccai G, et al. Esmolol reduces perioperative ischemia in noncardiac surgery: a meta-analysis of randomized controlled studies. J Cardiothorac Vasc Anesth. 2010;24:219-229. doi: 10.1053/j.jvca.2009.07.008.

30. OzawaY, Hayashi K, Kobori H. New Generation Calcium Channel Blockers in Hypertensive Treatment. Current hypertension reviews. 2006;2:103-111. doi: 10.2174/157340206776877370.

31. Peacock WF, Varon J, Baumann BM, et al. CLUE: a randomized comparative effectiveness trial of IV nicardipine versus labetalol use in the emergency department. Crit Care. 2011;15:R157. doi: 10.1186/cc10289.

32. Komura SN, Awad NI. The utility of bolus intravenous nicardipine for hypertensive emergencies in the ED. The American Journal of Emergency Medicine. 2016;34:2250.e12250.e3. doi:10.1016/j.ajem.2016.03.050.

33. Bergese SD, Puente EG. Clevidipine butyrate: a promising new drug for the management of acute hypertension. Expert Opin Pharmacother. 2010;11:281-295. doi: $10.1517 / 14656560903499293$.

34. Nguyen HM, Ma K, Pham DQ. Clevidipine for the treatment of severe hypertension in adults. Clin Ther. 2010;32:11-23. doi: 10.1016/j.clinthera.2010.01.025.

35. Aronson S, Dyke CM, Stierer KA, et al. The ECLIPSE trials: comparative studies of clevidipine to nitroglycerin, sodium nitroprusside, and nicardipine for acute hypertension treatment in cardiac surgery patients. Anesth Analg. 2008;107:1110-1121. doi: 10.1213/ane.ob013e31818240db.

36. Hottinger DG, Beebe DS, Kozhimannil T, Prielipp RC, Belani KG. Sodium nitroprusside in 2014: A clinical concepts review. Journal of Anaesthesiology, Clinical Pharmacology. 2014;30:462-471. doi: 10.4103/0970-9185.142799.

37. Immink RV, van den Born BJ, van Montfrans GA, et al. Cerebral hemodynamics during treatment with sodium nitroprusside versus labetalol in malignant hypertension. Hypertension. 2008;52:236-240. doi: 10.4103/0970-9185.142799.

38. Muiesan ML, Salvetti M, Amadoro V, et al. An update on hypertensive emergencies and urgencies. J Cardiovasc Med (Hagerstown). 2015;16:372-382. doi: 10.2459/ JCM.0000000000000223.

39. Sharma C, Soni A, Gupta A, Verma A, Verma S. Hydralazine vs nifedipine for acute hypertensive emergency in pregnancy: a randomized controlled trial. Am J Obstet Gynecol. 2017;217:687. e1-687.e6. doi: 10.1016 /j.ajog.2017.08.018.

40. Gillies MA, Kakar V, Parker RJ, et al. Fenoldopam to prevent acute kidney injury after major surgery-a systematic review and meta-analysis. Crit Care. 2015;19:449. doi:10.1186/ s13054-015-1166-4.

41. Ayaz SI, Sharkey CM, Kwiatkowski GM, et al. Intravenous enalaprilat for treatment of acute hypertensive heart failure in the emergency department. International Journal of Emergency Medicine. 2016;9:28. doi: 10.1186/s12245-0160125-4.

42. Tulman DB, Stawicki SPA, Papadimos TJ, Murphy CV, Bergese SD. Advances in Management of Acute Hypertension: A Concise Review. Discovery medicine. 2012;13:375-383. 
43. Gupta PK, Gupta H, Khoynezhad A. Hypertensive Emergency in Aortic Dissection and Thoracic Aortic Aneurysm - A Review of Management. Pharmaceuticals. 2009;2:66-76. doi: 10.3390/ph2030066.

44. Peacock WF, Chandra A, Char D, et al. Clevidipine in acute heart failure: Results of the A Study of BP Control in Acute Heart Failure-A Pilot Study (PRONTO). Am Heart J. 2014;167:529536. doi: 10.1016/ j.ahj.2013.12.023.

45. Kühl M, Lip GY, Varma C. Management of hypertension in relation to acute coronary syndromes and revascularisation. Curr Pharm Des. 2007;13:2556-2566.

46. Diercks DB, Ohman EM. Hypertension with acute coronary syndrome and heart failure. Ann Emerg Med. 2008;51:S34-36. doi: 10.1016/j.annemergmed.2007.11.012.

47. Peacock WF, Varon J, Ebrahimi R, Dunbar L, Pollack CV. Clevidipine for severe hypertension in patients with renal dysfunction: A VELOCITY trial analysis. Blood Press Suppl. 2011;1:20-25. doi: 10.3109/ 08037051.2010.539317.

48. Varon J, Soto-Ruiz KM, Baumann BM, et al. The management of acute hypertension in patients with renal dysfunction: labetalol or nicardipine? Postgrad Med. 2014;126:124-130. doi: 10.3810/pgm.2014.07.2790.

49. Olson-Chen C, Seligman NS. Hypertensive emergencies in pregnancy. Crit Care Clin. 2016;32:29-41. doi: 10.1016/j. ccc.2015.08.006.

50. Ahuja K, Charap MH. Management of perioperative hypertensive urgencies with parenteral medications. J Hosp Med. 2010;5:E11-16. doi: 10.1002/jhm.629.

51. Espinosa A, Ripollés-Melchor J, Casans-Francés R, et al. Perioperative Use of Clevidipine: A Systematic Review and Meta-Analysis. PLoS ONE. 2016;11:e0150625. doi: 10.1371/ journal.pone.0150625.

52. Kurnutala LN, Soghomonyan S, Bergese SD. Perioperative acute hypertension - role of Clevidipine butyrate. Frontiers in Pharmacology. 2014;5:197. doi: 10.3389/fphar.2014.00197.

53. Lenders JWM, Duh QY, Eisenhofer G, et al. Pheochromocytoma and Paraganglioma: An Endocrine Society Clinical Practice Guideline. J Clin Endocrinol Metab. 2014;99:1915-1942. doi:10.1210/jc.2014-1498.

54. Suzuki T, Eagle KA, Bossone E, Ballotta A, Froehlich JB, Isselbacher EM. Medical management in type B aortic dissection. Annals of Cardiothoracic Surgery. 2014;3:413-417. doi:10.3978/j.issn.2225-319X.2014.07.01.

55. Anderson CS, Huang Y, Wang JG, et al. Intensive BP reduction in acute cerebral haemorrhage trial (INTERACT): a randomized pilot trial. Lancet Neurol. 2008;7:391-399. doi: 10.1016/S14744422(08)70069-3.

56. Jauch EC, Saver JL, Adams HP Jr, et al. Guidelines for the early management of patients with acute ischemic stroke: a guideline for healthcare professionals from the American Heart Association/American Stroke Association. Stroke. 2013;44:870-947. doi: 10.1161/STR.ob013e318284056a.

57. Butcher KS, Jeerakathil T, Hill M, et al. The Intracerebral Hemorrhage Acutely Decreasing Arterial Pressure Trial. Stroke. 2013;44:620-626. doi: 10.1161/STROKEAHA.111.000188.

58. Ahmed N, Wahlgren N, Brainin $M$, et al. Relationship of $\mathrm{BP}$, antihypertensive therapy, and outcome in ischemic stroke treated with intravenous thrombolysis: retrospective analysis from Safe Implementation of Thrombolysis in Stroke-International Stroke Thrombolysis Register (SITS-ISTR). Stroke. 2009;40:2442-2449. doi: 10.1161/ STROKEAHA.109.548602.

59. He J, Zhang Y, Xu T, et al. Effects of immediate BP reduction on death and major disability in patients with acute ischemic stroke: the CATIS randomized clinical trial. JAMA. 2014;311:479-489. doi: 10.1001/jama.2013.282543. 